\title{
Magnetic control of homogeneous and dust-laden plasma jets*
}

\author{
Hideya Nishiyama† \\ Institute of Fluid Science, Tohoku University, 2-1-1, Katahira, Aoba-ku, Sendai, 980-8577, \\ Japan
}

\begin{abstract}
Magnetic control of plasma is considered to be one of the most effective method to utilize functions of plasma in the industrial applications. The objective of the present paper is to present the numerical modelings for homogeneous and dust-laden plasma jets in the applied magnetic field. The first model is for DC magnetic control of homogeneous plasma jet using three-fluid model. The second one is for RF inductive electromagnetic control of dust-laden plasma jet for turbulent or supersonic flow using Eulerian and Lagrangian approaches. Finally, future research to be considered is discussed in the numerical modeling for complex plasma jet under the electromagnetic field.
\end{abstract}

\section{INTRODUCTION}

Plasma is regarded as a multifunctional fluid, because it has high energy density, chemical reactivity, easy electromagnetic control, variable transport properties, elementary processes and strong luminescence [1]. Then it has been extensively utilized in the energy conversion system, material processing and environmental purification [2,3]. In these industrial applications it is very important to control a plasma jet precisely utilizing multifunctions of a plasma. Although there are many controllable parameters, such as discharge power, operating pressure, gas composition and flow rate, magnetic control of a plasma is considered to be one of the most effective method under the clean and stable conditions. There are some experimental papers concerning this method in the industrial applications [4-13]. However, as far as an author knows, there is very few theoretical papers focusing about the electromagnetic control of a plasma jet and a RF inductively coupled plasma taking into account the multifunctions in the numerical modeling [14-16].

In the present paper, two types of numerical modeling for magnetic control of homogeneous and dustladen plasma jets regarding plasma as a functional fluid is presented. The first numerical model is described for DC magnetic control of homogeneous, that is three component, plasma jet using a threefluid model which is applied for atom, ion and electron respectively. The second numerical model is also described for inductively coupled RF electromagnetic control of dust-laden plasma jets in two cases: one is turbulent dusty plasma jet [17], the other is supersonic dusty plasma jet $[18,19]$. The typical numerical results are shown here by computational realization. Finally, future research to be considered for complex modeling in magnetically controlled plasma jet is discussed, relating to the functions of plasma.

\section{MAGNETIC CONTROL OF HOMOGENEOUS PLASMA JET}

Plasma jet has been utilized in various kinds of material processings, propulsion system, MHD generation and environmental waste treatment. Therefore, it is very important to clarify the thermofluid characteristics of plasma species and collisional momentum and energy transfers controlled by DC magnetic field. For example, it was clarified experimentally that the plasma jet can be elongated to the

\footnotetext{
* Lecture presented at the 14th International Symposium on Plasma Chemistry, Prague, Czech Republic, 2-6 August 1999, pp. 1809-1918.

† Correspondence: E-mail: nishiyama@ifs.tohoku.ac.jp
} 
downstream and plasma parameters of electron number density and electron temperature can be changed by applying DC mirror type of magnetic field without changing other control parameters [10].

Furthermore, stabilization and suppression of unstable plasma jet behavior can be easily realized by applying magnetic field [12]. These magnetic control characteristics result from the utilization of Lorentz force, Joule heating, variable transport properties and further collision process between plasma species. In the present section, the thermofluid characteristics of ion, electron and atom in the applied DC mirror type magnetic field is clarified numerically using three-fluid model [14].

Figure 1 shows a schematic model of homogeneous plasma jet in the applied DC magnetic field. A plasma jet is issued into a tube and two solenoidal coils are set at the nozzle exit to produce a mirror type magnetic field. To utilize the hall effect in a plasma jet, the swirl flow is also given at the nozzle exit crossing perpendicularly to the magnetic lines force.
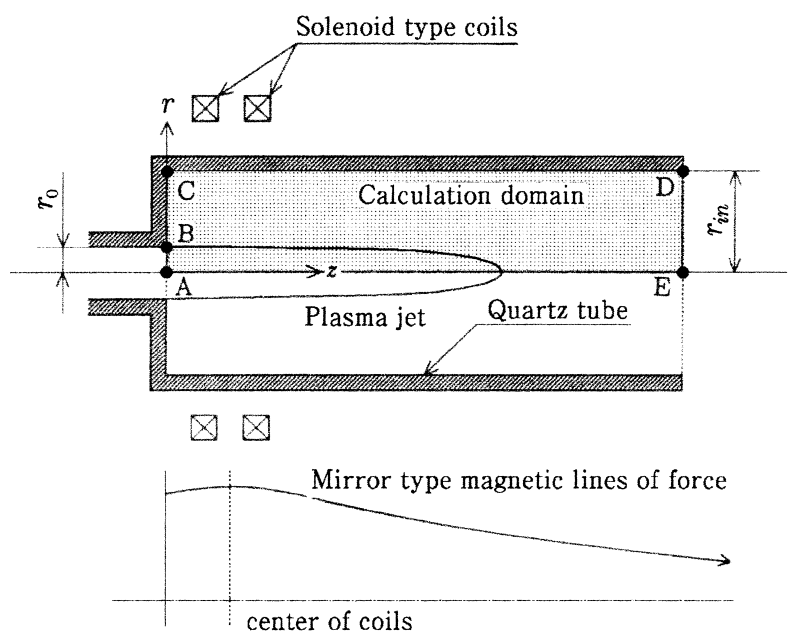

Fig. 1 Schematic model of homogeneous plasma jet in the applied DC magnetic field.

In order to derive governing equations, the following assumptions are introduced here.

1 Working fluid is argon gas, and plasma is regarded as an ideal gas and continuum.

2 Jet is axisymmetric subsonic laminar flow with swirl.

3 Viscous dissipation in energy equations is neglected.

4 Plasma is optically thin, and for elementary processes, only two-body collisional ionization and three-body recombination are considered.

5 Self induced magnetic field is negligibly small, and applied magnetic field is given by Biot-Savart's formula.

6 Transport properties have temperature and pressure dependence and anistropy.

7 Mass difference between argon atom and argon ion is neglected.

8 Pressure gradient current of electron in Ohm's law is neglected.

9 As an ideal condition, velocity and temperature of each plasma species are equal each other in equilibrium condition at the nozzle exit.

According to the above assumption, governing equations for atom, ion and electron in a plasma jet are derived using three-fluid model.

Governing equations

- Conservation of mass

Net production of electrons by two-body collisional ionization and three-body recombination

- Conservation of momentum 
—Lorentz force

- Collisional momentum transfer

-Momentum transfer by species production

- Conservation of energy

—Joule heating

-Collisional energy transfer

-Ionization-recombination energy transfer

-Energy transfer by species production

-Radiation loss

- Ohm's law

- Biot-Savart's formula

- Equation of state

Figure 2 shows the isocontours of temperature and velocity for each plasma species with and without DC magnetic field. In the region from the jet fringe towards the wall thermal nonequilibrium of $T_{e}>T_{i} \cong T_{n}$ is observed due to the production of Joule heating and the poor collision between plasma species. All the temperatures increase outer from the jet fringe by applying the DC magnetic field. The electron velocity is 1.2 times faster than the heavy particle velocities due to the small mass and small viscosity of electron, and further it is changed considerably in the central region by Lorentz force in the applied DC magnetic field. The ion velocity is a little faster than the atom velocity just after nozzle exit due to the viscosity difference and the induction electric field. However, they become almost the same in the downstream and both are hardly influenced by the DC magnetic field.
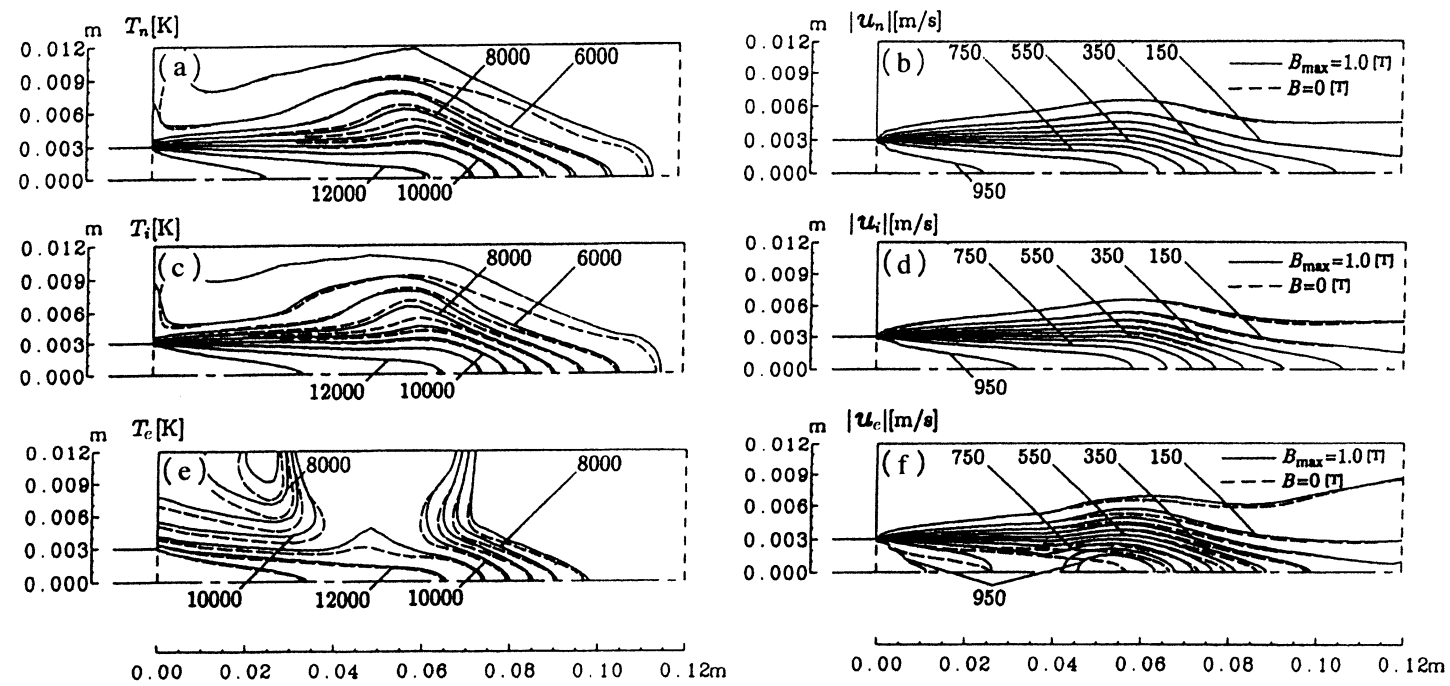

Fig. 2 Isocontours of temperature and velocity of plasma species with and without DC magnetic field.

Figure 3 shows the axial variation of three components of Lorentz force acted on a plasma jet in the applied DC magnetic field. The radial component of Lorentz force given by $F_{r}=J_{\theta} \times B_{z}$ is strongest, which plays a diverging or a converging effect on a plasma jet. It is clearly shown that the fluid appears to behave predominantly as a magnetohydrodynamic fluid up to about $50 \mathrm{~mm}$.

Figure 4 shows the variation of the energy transfer between plasma species with the magnetic field intensity. The energy transfer is little influenced by the magnetic field intensity, but the one between electron and the heavy particles increases at first even in a small magnetic field intensity. This is because under even a small magnetic field intensity, electrons are trapped in the central region and then the number density and collision frequency increase. 


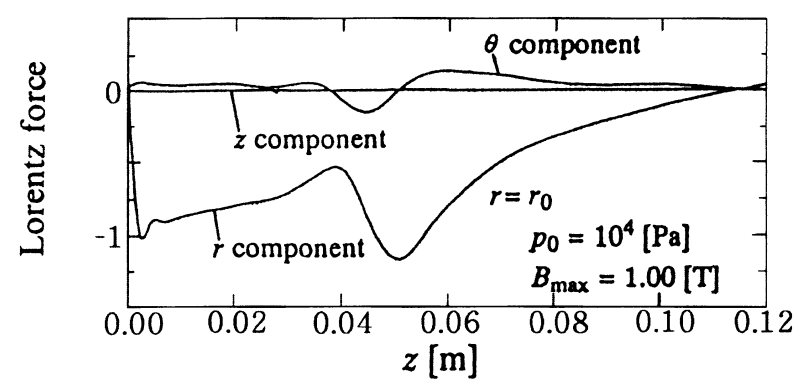

Fig. 3 Axial variation of Lorentz force.
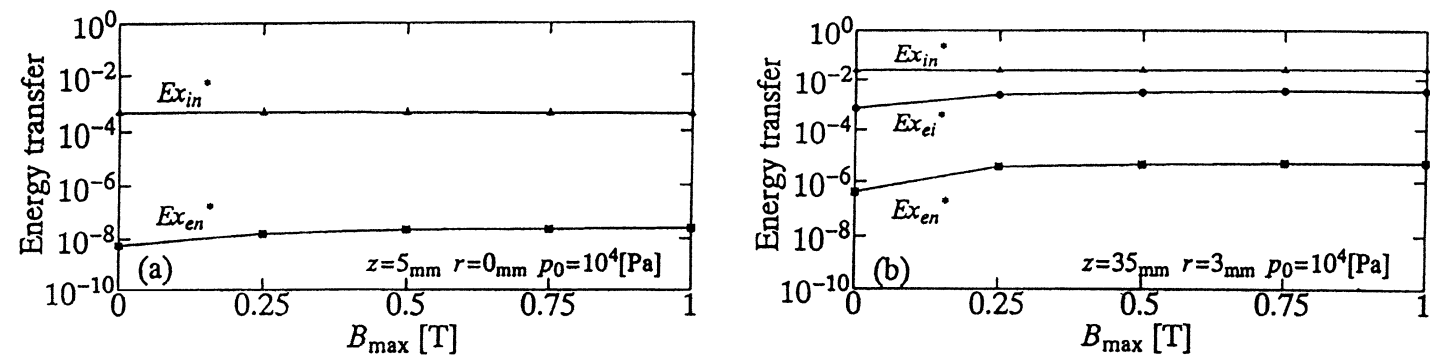

Fig. 4 Energy transfer between each plasma species with the magnetic field.

\section{RF INDUCTIVE ELECTROMAGNETIC CONTROL OF DUST-LADEN PLASMA JET}

A plasma spraying has been extensively utilized to produce a functional coating and to treat a surface modification [20,21]. In this processing there are many controllable parameters which are accompanied by complex thermofluid phenomena in extreme conditions. It is very useful to investigate the possibility to control the plasma jet directly and then to control the particle characteristics indirectly by applying the magnetic field. In the present section, the main objective is to clarify the control characteristics of thermofluid field of plasma jet and of particle characteristics such as particle trajectory, particle velocity and particle phase change by RF inductive electromagnetic field. There are described for two cases, one is for turbulent dusty plasma jet [17] and the other is for supersonic dusty plasma jet [18,19]. In order to derive the governing equations the following assumptions are introduced here.

1 The plasma is continuous and ideal gas in LTE and optically thin;

2 The flow, temperature and electromagnetic fields are axisymmetric 2D.

3 The injected particles are spherical with same size and the internal temperature is uniform in low $B i$ number.

4 Only drag force is acted on the particle.

5 Collision between particles is neglected under the dilute particle-loading condition.

6 There is one-way coupling between the dispersed phase and the carrier phase.

7 The nozzle and the chamber are electrically insulated.

The key points of governing equations for plasma jet, particle and induction electromagnetic field are respectively as follows.

Governing equations

- Plasma jet

Case 1: Turbulent flow $(k-\epsilon$ model)

Case 2: Low pressure supersonic flow

Eulerian approach

-Variable thermodynamic and transport properties 
- Lorentz force

—Joule heating

-Plasma-substrate interaction

- Particle

Lagrangian approach

-Plasma-particle interaction (one-way coupling)

-Phase change criterion

- Variable thermodynamic and transport properties in a particle boundary layer

- $\quad$ RF induction electromagnetic field

Maxwell's equation (vector potential)

Ohm's law

\section{Turbulent dusty plasma jet}

Figure 5 shows a schematic model of turbulent dusty plasma jet imposed RF coil on the reactor. The effect of induction RF heating on plasma flow and particle temperature are described here [17].

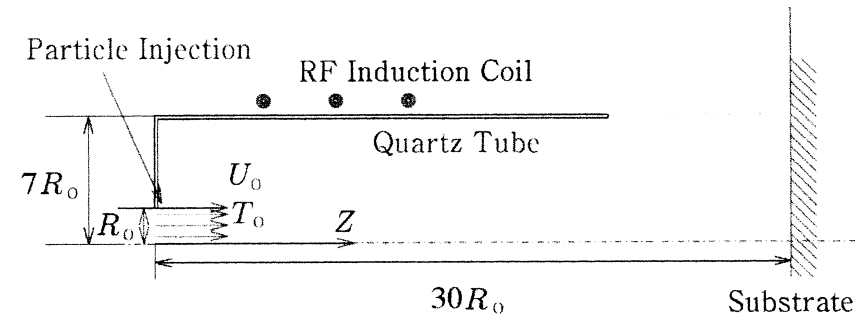

Fig. 5 Schematic model of turbulent dusty plasma jet in the RF inductive electromagnetic field.

Figure 6 shows the axial distributions of magnetic field intensity for four types of coil current direction. The maximum magnetic intensities of cusp types (types 2-4) are smaller than that of mirror type (type 1) under the same coil electric current. Therefore the induction magnetic field intensity and distribution can be controlled by changing the direction of the levitation coil current independently.

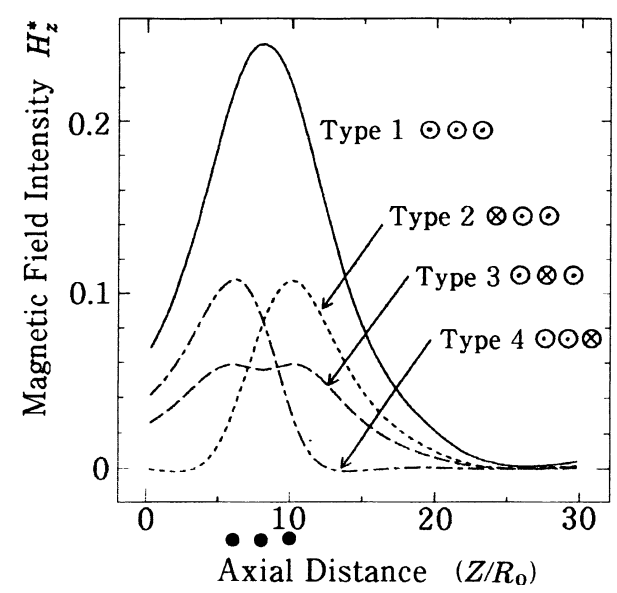

Fig. 6 Axial magnetic field distribution.

Figure 7 shows the component of Joule heating in type 1 and type 2 for comparison. In type 2, the sunken area is observed due to the cancelling of a reversed current direction of the first coil to the second and the third ones. The location of maximum Joule heating is moved relatively to the downstream side 
compared with that of type 1 corresponding to the distribution of magnetic field intensity as shown in Fig. 6.

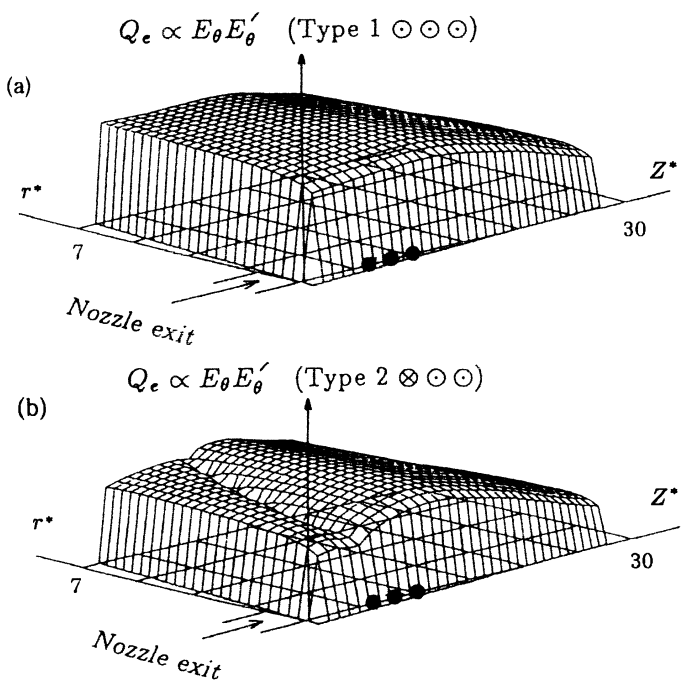

Fig. 7 Joule heating distribution.

Figure 8 shows the isocontours of plasma temperature which is influenced by Joule heating. Compared with type 1 , the high temperature region in type 2 is reduced and is shifted to the downstream corresponding to the Joule heating characteristics as shown in Fig. 7. It is shown that the plasma temperature field can be controlled for each purpose by changing the coil current directions.

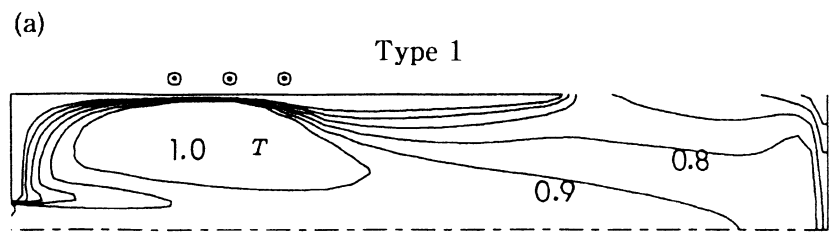

(b)

Type 2

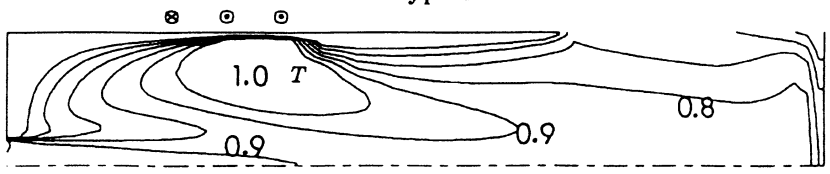

Fig. 8 Isocontours of plasma temperature field.

Figure 9 shows the dispersed features of tungsten particles with different ranges of particle temperature in both the cases without RF coil (a) and type 1 (b) respectively. In the case of the type 1 with inclined particle injection, the particles are dispersed more broadly in the radial direction and they penetrate into the core region of the jet due to the reactive effect of the Lorentz force through the jet. The particles are rapidly heated up to the boiling point because of the longer residence time and wider high temperature region resulting from the inductive electromagnetic effects.

\section{Supersonic dusty plasma jet}

Figure 10 shows a schematic model of the electromagnetically controlled virtual plasma spraying system $[18,19]$. A virtual low pressure plasma spraying is conducted to clarify the effects of induction RF electromagnetic field, particle diameter, particle injection velocity and nozzle angle on the plasma jet and in-flight particle characteristics here. 
(a)
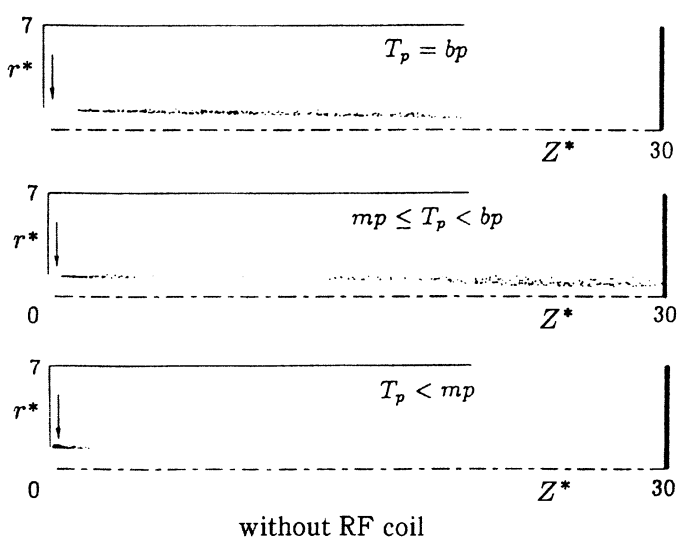

Fig. $9 \mathrm{~W}$ Particle temperature and its dispersion. (b)
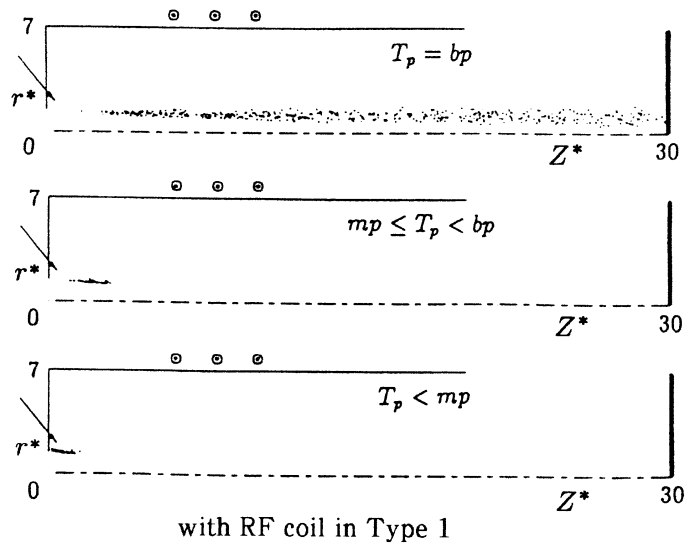

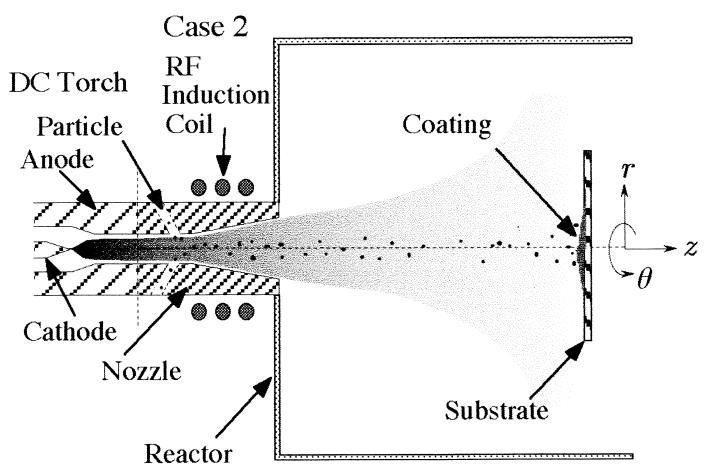

Fig. 10 Schematic model of the electromagnetically controlled virtual plasma spraying system.

Figure 11 shows the isocontours of plasma temperature without and with RF coil imposed on the nozzle. In the case 2 , the plasma temperature increases considerably from the nozzle region to just downstream in the reactor compared with that in case 1. This is because of the active induction Joule heating in the nozzle by applying the RF induction coil. It is expected that the particle can be heated up rapidly in the initial stage of the reactor.

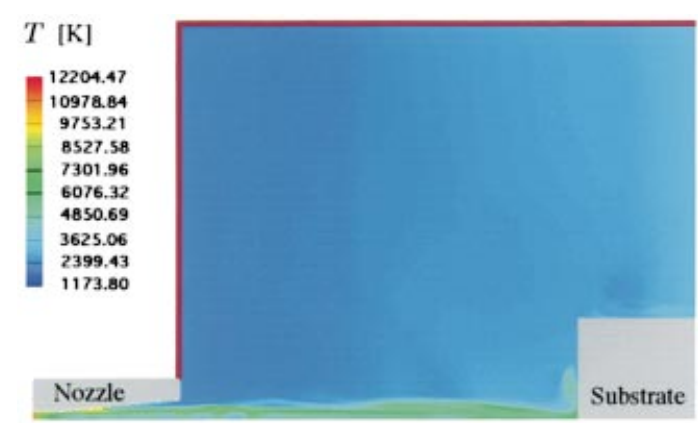

Casel (without RF coil)

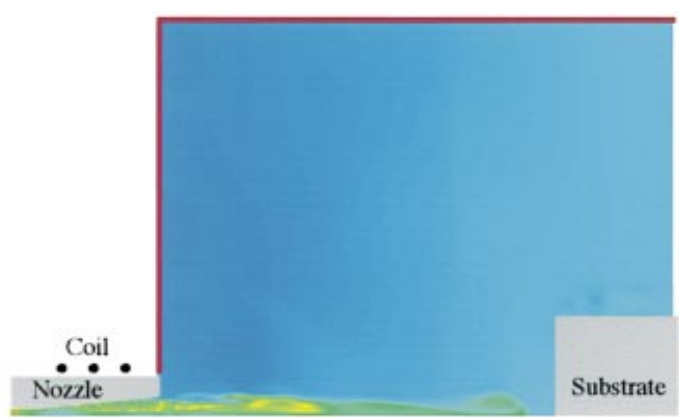

Case2 (with RF coil)

Fig. 11 Isocontours of plasma temperature field. 
Figure 12 shows the effect of the particle injection nozzle angle on the particle trajectory with and without RF coil. It is clearly seen that the $20 \mu \mathrm{m}$ particle spreads more outward with the increase in the nozzle angle, although the flow separation occurs just upstream from the nozzle exit at $\alpha=8.9^{\circ}$. When the RF coil is imposed, the particle spreads outward a little for all nozzle angles. It is shown that the nozzle angle is the important parameter and the application of RF coil is second effect on the particle trajectory.

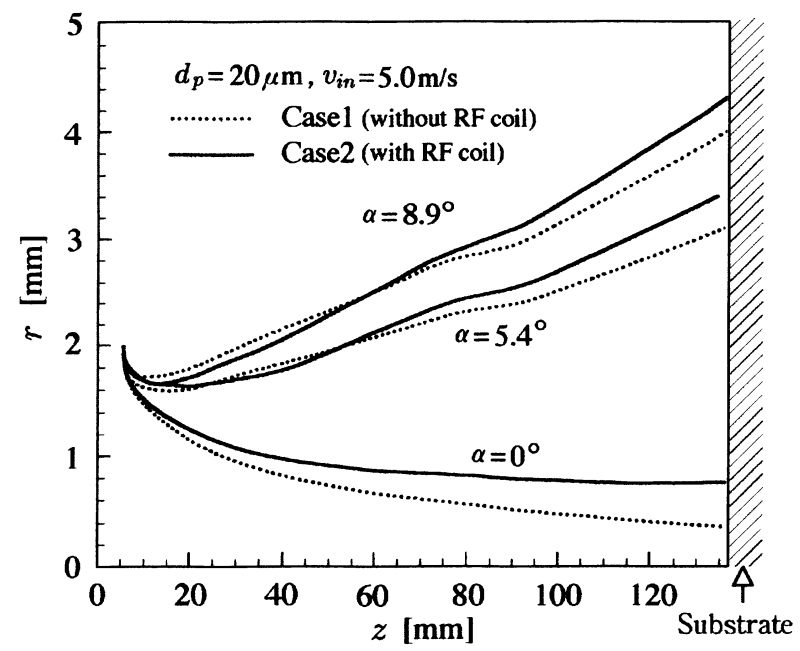

Fig. 12 Effect of nozzle angle on the particle trajectory.

Figure 13 shows the optimum range between the particle diameter and the particle injection velocity for fully molten state of in-flight particle. The range of particle diameter becomes wider for smaller particle injection velocity. When the RF magnetic field is inductive in the case 2 , the range of particle diameter for fully molten state also becomes wider, showing that even larger particle can be easily melted in a short residence time.

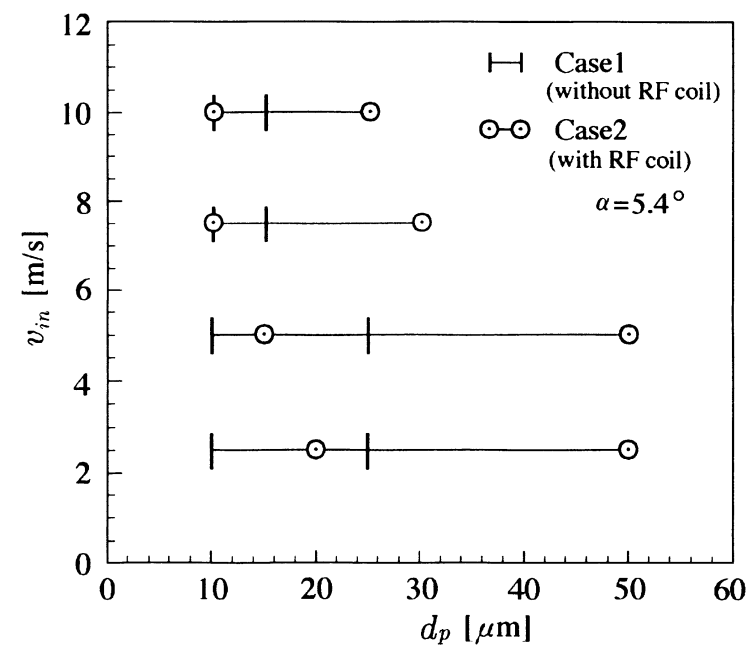

Fig. 13 Correlation between the particle diameter and the particle injection velocity for fully molten state.

\section{CONCLUSION}

The new numerical models are described for the homogeneous and dust-laden plasma jets in the applied DC magnetic field or RF induction electromagnetic field from MHD and multiphase flow approaches 
taking into account the individual functions of plasma. The results obtained by the computational realization are as follows:

1 The application of DC magnetic field utilizing Hall effect is most effective to control the electron velocity and temperature in a homogeneous plasma jet.

2 The induction of RF electromagnetic field is most effective to in-flight particle heating utilizing Joule heating in a plasma jet, but is a second effect on particle velocity and dispersion.

It is very important to solve the reverse problem for optimum magnetic field distribution and intensity to control a complex plasma jet effectively. For future research, it is also very interesting to investigate following factors: 3D case, complex turbulence structure with particles, particle behavior such as particle cluster, particle shape, particle charging and particle impact process, transport properties taking into account thermal diffusion of mixing gas with chemical reaction, particle evaporation and nonequilibrium effect etc.

\section{ACKNOWLEDGEMENT}

These works were partly supported by the Ministry of Education, Science, Sports and Culture in Japan. The numerical simulation was conducted using a Cray C-916 at the Super Computer Center of the Institute of Fluid Science, Tohoku University.

\section{REFERENCES}

1 H. Nishiyama. J. Machine Sci. 44, 193-198 (1992), in Japanese.

2 E. Pfender. Pure Appl. Chem. 60(5), 591-606 (1988).

3 M. I. Boulos. IEEE Trans. Plasma Sci. 19(6), 1078-1089 (1991).

4 F. H. Coensgen, A. E. Sherman, W. E. Nexsen, W. F. Cummius. Phys. Fluids 3(5), 764-768 (1960).

5 K. Kuriki, O. Okada. Phys. Fluids 13(9), 2262-2269 (1990).

6 H. Fujita, Y. Okuno, S. Yagara. J. Appl. Phys. 67(10), 6114-6117 (1990).

7 H. Takeda. J. High Temp. 16, 357-367 (1990).

8 W. K. Kim, K. W. Whang. Mater. Manufact. Process. 8(1), 83-98 (1993).

9 K. Taniguchi, N. Fukumoto, M. Nagata, T. Uyama, N. Eguchi, A. Ohmori. J. Jap. Therm. Spraying Soc. 36(1), 81-87 (1996).

10 H. Nishiyama, T. Sato, S. Kamiyama. Proc. of 10th Int. Conf. on Gas Discharges and Their Applications 2 , 778-781 (1992).

11 H. Nishiyama, T. Sato, A. Veefkind, S. Kamiyama. Heat Mass Transfer 30(5), 291-296 (1995).

12 T. Sato, H. Nishiyama, S. Kamiyama. Proc. of 12th Intern. Symp. on Plasma Chem. Minneapolis, 3, 1779-1784 (1995).

13 H. Nishiyama, H. Tsukada, Y. Matsushima, S. Kamiyama. J. Phys. D: Appl. Phys. 30(20), 2804-2811 (1997).

14 H. Nishiyama, T. Saito, S. Kamiyama. Plasma Chem. Plasma Process. 16(2), 265-286 (1996).

15 H. Nishiyama, Y. Muro, S. Kamiyama. J. Phys. D: Appl. Phys. 29(10), 2634-2643 (1996).

16 H. Nishiyama, Y. Fukai, S. Kamiyama. Jap. Soc. Mech. Eng. Intern. J. Ser. B 41(2), 502-510 (1998).

17 S. W. Nam, H. Nishiyama, S. Kamiyama. Jap. Soc. Mech. Eng. Intern. J. Ser. B 39(1), 134-140 (1996).

18 H. Nishiyama, M. Kuzuhara, O. P. Solonenko, S. Kamiyama. Plasma Chem. Plasma Process. 19(3), 363-381 (1999).

19 H. Nishiyama, M. Kuzuhara, M. Shiozawa, T. Sato, O. P. Solonenko. Thermal Plasma Torches and Technolo. O.P. Solonenko, ed). Vol. 2, pp. 41-54. Cambridge International Science Publications, Cambridge, UK (1999).

20 R. B. Heinmann. Plasma-Spray Coating, VCH Publishers (1996).

21 O. P. Solonenko, M. F. Zhukov. Thermal Plasma New Material Technology, Vol. 2. Cambridge International Science Publications, Cambridge, UK (1995). 